\title{
THE LOWER RANK OF DIRECT PRODUCTS OF HEREDITARILY JUST INFINITE GROUPS
}

\author{
BENJAMIN KLOPSCH AND MATTEO VANNACCI
}

\begin{abstract}
We determine the lower rank of the direct product of finitely many hereditarily just infinite profinite groups of finite lower rank.
\end{abstract}

\section{INTRODUCTION}

For primes $p$, the theory of $p$-adic analytic pro- $p$ groups plays a central part in the study of pro- $p$ groups and has interesting applications in infinite group theory; see [6, 2]. According to a well-known algebraic characterisation, a pro- $p$ group $G$ is $p$-adic analytic if and only if $G$ has an open subgroup of finite rank. Here the rank of $G$ is defined as

$$
\operatorname{rk}(G)=\sup \left\{\mathrm{d}(H) \mid H \leq_{\mathrm{o}} G\right\},
$$

where $\mathrm{d}(H)$ denotes the minimal number of topological generators of the open subgroup $H \leq_{\mathrm{o}} G$. Indeed, Lubotzky and Mann [7] even established the following refinement: a pro- $p$ group $G$ is $p$-adic analytic if and only if the upper $\operatorname{rank} \bar{L}_{\mathrm{d}}(G)=\lim \sup \left\{\mathrm{d}(H) \mid H \leq_{\mathrm{o}} G\right\}$ is finite, where the limit superior is taken over the net of open subgroups ordered by reverse inclusion; moreover, in this case $\bar{L}_{\mathrm{d}}(G)$ is equal to the dimension $\operatorname{dim}(G)$ of $G$ as a $p$-adic manifold.

Lubotzky and Mann also introduced, for a general profinite group $G$ the lower rank

$$
\operatorname{lr}(G)=\underline{L}_{\mathrm{d}}(G)=\liminf \left\{\mathrm{d}(H) \mid H \leq_{\mathrm{o}} G\right\},
$$

where again the limit inferior is taken over the net of open subgroups ordered by reverse inclusion. They proved that the lower rank of a compact $p$-adic analytic group coincides with the number of generators of its associated $\mathbb{Q}_{p}$-Lie algebra. By a classical theorem of Kuranishi [5], this implies that the lower rank of any compact $p$-adic analytic group with semi-simple $\mathbb{Q}_{p}$-Lie algebra is equal to 2 .

Lubotzky and Shalev [8] continued the study of the lower rank and showed, for instance, that there exist non-analytic pro- $p$ groups of finite lower rank. Refining their techniques, Barnea [1] established, for instance, that the lower rank of the $\mathbb{F}_{p} \llbracket t \rrbracket$-analytic group $\mathrm{SL}_{2}\left(\mathbb{F}_{p}[[t]]\right)$ is

2010 Mathematics Subject Classification. Primary 20E18; Secondary 20F06.

Key words and phrases. Lower rank, just infinite group, profinite group, direct product. 
equal to 2. Computing the lower ranks of profinite groups is usually rather challenging and producing new families of groups of finite lower rank is of considerable interest.

In this paper we are interested in the lower ranks of finite direct products of hereditarily just infinite profinite groups. We recall that a profinite group $G$ is just infinite, if $G$ is infinite and every non-trivial closed normal subgroup $N \unlhd_{\mathrm{c}} G$ is open in $G$. The group $G$ is hereditarily just infinite if every open subgroup $H \leq_{\mathrm{o}} G$ is just infinite.

There are many non-(virtually abelian) hereditarily just infinite profinite groups of finite lower rank, including those that are $p$-adic analytic; cf. 44. However, Ershov and Jaikin-Zapirain proved that there exist also hereditarily just infinite pro- $p$ groups of infinite lower rank; see [3, Corollary 8.10]. More recently, the second author of the present paper analysed the lower rank in a family of non-(virtually pro- $p$ ) hereditarily just infinite groups and conjectured that there exist such groups of any given lower rank in $\mathbb{N}_{\geq 2} \cup\{\infty\}$; see [10]. We establish the following theorem and corollary.

Theorem A. Let $G=\prod_{i=1}^{n} G_{i}$ be a non-trivial direct product of finitely many hereditarily just infinite profinite groups of finite lower rank. Set

$$
d=\max \left\{\operatorname{lr}\left(G_{i}\right) \mid 1 \leq i \leq n\right\} \quad \text { and } r=\max \left\{r_{p} \mid p \text { prime }\right\},
$$

where $r_{p}$ denotes the number of indices $i$ such that $G_{i}$ is virtually- $\mathbb{Z}_{p}$. Then the lower rank of $G$ is $\operatorname{lr}(G)=\max \{d, r\}$.

Corollary B. The non-trivial direct product $G=\prod_{i=1}^{n} G_{i}$ of finitely many pairwise non-commensurable hereditarily just infinite profinite groups of finite lower rank has lower rank

$$
\operatorname{lr}(G)=\max \left\{\operatorname{lr}\left(G_{i}\right) \mid 1 \leq i \leq n\right\} .
$$

In the proof we use basic facts about the structure of just infinite profinite groups, in particular a result of Reid [9]. We emphasise that, in general, the lower rank of a direct product of profinite groups can be as large as the sum of the lower ranks of the factors. For instance, the lower rank of a free abelian pro- $p$ group $\mathbb{Z}_{p} \times \ldots \times \mathbb{Z}_{p}$, with $n$ factors, is clearly $n$.

Theorem A can be regarded as a generalisation of the aforementioned result, due to Kuranishi, Lubotzky and Mann, that every compact $p$-adic analytic group with semi-simple $\mathbb{Q}_{p}$-Lie algebra has lower rank 2 . Finally, we remark that Theorem $\mathrm{A}$ can be applied to the family of hereditarily just infinite profinite groups of finite lower rank described in [10]. In this way we obtain many new examples of profinite groups of finite lower rank.

\section{Preliminaries}

Clearly, profinite groups of finite lower rank are finitely generated and thus countably based. Restricting to the latter class of profinite 
groups, we can navigate around the general notion of the limit inferior of a net. The lower rank of a countably based profinite group $G$ is

$$
\begin{array}{r}
\operatorname{lr}(G)=\min \left\{\sup \left\{\inf \left\{\mathrm{d}\left(H_{i}\right) \mid i \geq N\right\} \mid N \in \mathbb{N}\right\} \mid\left(H_{i}\right)_{i \in \mathbb{N}} \in \mathcal{C}(G)\right\} \\
\in \mathbb{N}_{0} \cup\{\infty\},
\end{array}
$$

where

$$
\mathcal{C}(G)=\left\{\left(H_{i}\right)_{i \in \mathbb{N}} \mid G=H_{1_{\mathrm{o}}} \geq H_{2_{\mathrm{o}}} \geq \ldots \text { and } \bigcap_{i \in \mathbb{N}} H_{i}=1\right\}
$$

is the collection of all descending chains of open subgroups of $G$ that form a neighbourhood base for the identity element.

In other words, a countably based profinite group $G$ has lower rank at most $r$ if there exists a descending chain of $r$-generated open subgroups of $G$ that form a neighbourhood base for the identity.

In preparation for the proof of Theorem $\mathrm{A}$ we collect two basic lemmata. Recall that a profinite group $G$ possesses virtually a grouptheoretic property $\mathfrak{P}$ if $G$ has an open subgroup $H$ that has $\mathfrak{P}$. We abbreviate "virtually-(infinite procyclic pro- $p$ )" to "virtually- $\mathbb{Z}_{p}$ ".

Lemma 2.1. Let $G$ be a virtually abelian, hereditarily just infinite profinite group. Then $G$ is virtually- $\mathbb{Z}_{p}$ for a suitable prime $p$.

Proof. Let $A$ be an open abelian subgroup of $G$. As $A$ is just infinite, it is a pro- $p$ group for some prime $p$ and infinite pro-cyclic. Thus $A$ is isomorphic to $\mathbb{Z}_{p}$.

Lemma 2.2. Let $H$ be a just infinite profinite group that is not virtually abelian, and let $L \leq_{0} H$. Then there exists $x \in H$ such that $L$ is not contained in $\mathrm{C}_{H}(x)$.

Proof. For a contradiction, assume that $L \subseteq \mathrm{C}_{H}(x)$ for all $x \in H$. Then $L$ is contained in the centre $\mathrm{Z}(H)=1$, hence $L=1$.

\section{Proof of Theorem $\mathrm{A}$}

Before proving Theorem $\mathrm{A}$ we establish another auxiliary result.

Lemma 3.1. Let $n \in \mathbb{N}$ and let $G=\prod_{i=1}^{n} G_{i}$ be a direct product of finitely many hereditarily just infinite profinite groups of finite lower rank, where none of them is virtually abelian, and define the integer $d=\max \left\{\operatorname{lr}\left(G_{i}\right) \mid 1 \leq i \leq n\right\}$. Then for every basic open neighbourhood $\prod_{i=1}^{n} U_{i}$ of the identity element in $G$, with $U_{i} \subseteq_{\mathrm{o}} G_{i}$, there exist open subgroups $H_{i} \leq_{\mathrm{o}} G_{i}$, for $1 \leq i \leq n$, such that

(1) $H_{i} \subseteq U_{i}$ and $\mathrm{d}\left(H_{i}\right) \leq d$ for $1 \leq i \leq n$,

(2) $H_{i} ¥ H_{j}$ for $1 \leq i<j \leq n$.

Proof. For each $i \in\{1, \ldots, n\}$, the group $G_{i}$ admits a descending chain of open $d$-generated subgroups $H_{i, 1} \supsetneqq H_{i, 2} \supsetneqq \ldots$ satisfying $H_{i, k} \subseteq U_{i}$ for $k \in \mathbb{N}$. By [9, Theorem E], a non-(virtually abelian) just infinite 
profinite group does not contain any proper open subgroups isomorphic to the whole group. Hence, for $1 \leq i \leq n$, the groups $H_{i, k}, k \in \mathbb{N}$, are pairwise non-isomorphic. Consequently there are $k_{1}, \ldots, k_{n} \in \mathbb{N}$ such that $H_{1}=H_{1, k_{1}}, \ldots, H_{n}=H_{n, k_{n}}$ are pairwise non-isomorphic.

Now, let $G=\prod_{i=1}^{n} G_{i}$ be a direct product of finitely many hereditarily just infinite profinite groups of finite lower rank. We set

$$
\ell=\max \{d, r\}
$$

where $d=\max \left\{\operatorname{lr}\left(G_{i}\right) \mid 1 \leq i \leq n\right\}$ and $r=\max \left\{r_{p} \mid p\right.$ prime $\}$ are defined as in the statement of Theorem $\mathrm{A}$, here $r_{p}$ denotes the number of $i \in\{1, \ldots, n\}$ such that $G_{i}$ is virtually- $\mathbb{Z}_{p}$.

Clearly $\operatorname{lr}(G) \geq \ell$. Let $U=\prod_{i=1}^{n} U_{i}$ be a basic open neighbourhood of the identity element in $G$, with $U_{i} \subseteq \subseteq_{\mathrm{o}} G_{i}$. We need to find an $\ell$-generated open subgroup $K \leq_{\mathrm{o}} G$ with $K \subseteq U$.

Without loss of generality, the first $m$ factors $G_{1}, \ldots, G_{m}$ are not virtually abelian, while the remaining $n-m$ factors $G_{m+1}, \ldots, G_{n}$ are virtually abelian. By Lemma 2.1, there exists, for each $i \in\{m+1, \ldots, n\}$, a prime $q_{i}$ such that $G_{i}$ is virtually- $\mathbb{Z}_{q_{i}}$. Reordering the factors and descending to an appropriate open subgroup $C \leq_{\mathrm{o}} \prod_{i=m+1}^{n} G_{i}$ we can arrange that $C \subseteq \prod_{i=m+1}^{n} U_{i}$ and

$$
C=C_{1} \times \ldots \times C_{s}, \quad \text { with } C_{i}=\overline{\left\langle y_{i 1}, \ldots, y_{i t_{i}}\right\rangle} \cong \mathbb{Z}_{p_{i}}^{t_{i}} \text { for } 1 \leq i \leq s,
$$

where $s \in \mathbb{N} \cup\{0\}$ with $s \leq n-m$, the positive integers $t_{i}=r_{p_{i}}$ satisfy $\sum_{i=1}^{s} t_{i}=n-m$ and $p_{1}, \ldots, p_{s}$ denote distinct primes. It is convenient to set $y_{i j}=1$ for $1 \leq i \leq s$ and $t_{i}+1 \leq j \leq \ell$ as well as for $s+1 \leq i \leq n$ and $1 \leq j \leq \ell$. We now work in the open subgroup $\prod_{i=1}^{m} G_{i} \times C \leq_{\mathrm{o}} G$.

By Lemma 3.1, we can choose subgroups $H_{i} \leq_{\mathrm{o}} G_{i}$ with generators $h_{i 1}, \ldots, h_{i d}$, for $1 \leq i \leq m$, such that

$$
H_{i}=\overline{\left\langle h_{i 1}, \ldots, h_{i d}\right\rangle} \subseteq U_{i} \quad \text { and } \quad H_{i} ¥ H_{j} \quad \text { for } 1 \leq i<j \leq m .
$$

Again it is convenient to set $h_{i j}=1$ for $1 \leq i \leq m$ and $d+1 \leq j \leq \ell$ as well as for $m+1 \leq i \leq n$ and $1 \leq j \leq \ell$. We write $H=\prod_{i=1}^{m} H_{i}$ for the internal direct product of $H_{1}, \ldots, H_{m}$.

To conclude the proof, it suffices to produce a $\ell$-generated open subgroup $K \leq_{\mathrm{o}} H \times C \leq_{\mathrm{o}} G$. We consider

$$
K=\overline{\left\langle g_{1}, g_{2}, \ldots, g_{\ell}\right\rangle} \leq_{\mathrm{c}} H_{1} \times \cdots \times H_{m} \times C=H \times C,
$$

where $g_{i}=h_{i} y_{i}$ with

$$
h_{i}=h_{1 i} h_{2 i} \cdots h_{n i} \in H \quad \text { and } \quad y_{i}=y_{1 i} y_{2 i} \cdots y_{n i} \in C \quad \text { for } 1 \leq i \leq \ell .
$$

Clearly, $K$ is $\ell$-generated. Furthermore, $K$ is a sub-direct product of $H_{1}, \ldots, H_{m}$ and $C$, i.e., it is a closed subgroup of $H_{1} \times \ldots \times H_{m} \times C$ that projects onto each of the $m+1$ direct factors. The proof of Theorem $\mathrm{A}$ can therefore be completed by appealing to the next proposition, which is also of independent interest. 
Proposition 3.2. Let $m \in \mathbb{N}_{0}$, and let

$$
K \leq_{\mathrm{c}} H_{1} \times \ldots \times H_{m} \times C
$$

be a sub-direct product of $m$ pairwise non-isomorphic finitely generated just infinite profinite groups $H_{1}, \ldots, H_{m}$ that are not virtually abelian and a finitely generated abelian profinite group $C$.

Then $K$ is an open subgroup of $\prod_{i=1}^{m} H_{i} \times C$.

Proof. We may assume that $m \geq 1$. Put $H=\prod_{i=1}^{m} H_{i}$. For each $j \in\{1, \ldots, m\}$, let $\pi_{j}: H \times C \rightarrow H_{j}$ denote the projection onto $H_{j}$; and let $\pi_{C}: H \times C \rightarrow C$ denote the projection onto $C$. Fix finitely many generators $h_{1}, \ldots, h_{d}$ for $H_{1}$ and $y_{1}, \ldots, y_{s}$ for $C$ so that

$$
H_{1}=\overline{\left\langle h_{1}, \ldots, h_{d}\right\rangle} \text { and } C=\overline{\left\langle y_{1}, \ldots, y_{s}\right\rangle} \text {. }
$$

Since $K$ is a sub-direct product, we find $h_{1}^{*}, \ldots, h_{d}^{*}, y_{1}^{*}, \ldots, y_{d}^{*} \in K$ such that $h_{i}^{*} \pi_{1}=h_{i}$ for $1 \leq i \leq d$ and $y_{j}^{*} \pi_{C}=y_{j}$ for $1 \leq j \leq s$.

We observe that it suffices to show that, for each $j \in\{1, \ldots, m\}$, there exist an open subgroup $K_{j} \leq_{\mathrm{o}} H_{j}$ with $K_{j} \leq K$. For then we get $\widetilde{K}=K_{1} \times \cdots \times K_{m} \leq_{\mathrm{o}} H$ and, setting $N=|H: \widetilde{K}|$ !, we obtain $h^{N} \in \bigcap\left\{\widetilde{K}^{g} \mid g \in H\right\} \subseteq \widetilde{K}$ for every $h \in H$. As $C$ is central, this implies $y_{i}^{N}=\left(y_{i}^{*}\right)^{N}\left(\left(y_{i}^{*}\right)^{-1} y_{i}\right)^{N} \in K$ for $1 \leq i \leq s$, and we deduce from $\widetilde{K} \times \overline{\left\langle y_{1}^{N}, \ldots, y_{s}^{N}\right\rangle} \leq_{\mathrm{o}} H \times C$ that $K \leq_{\mathrm{o}} H \times C$.

It remains to construct the aforementioned subgroups $K_{j} \leq_{\mathrm{o}} H_{j}$ with $K_{j} \leq K$ for $1 \leq j \leq m$. By symmetry, it is enough to manufacture $K_{1}$. Indeed, we construct recursively, for each $1 \leq i \leq m$, a subgroup $K^{(i)} \leq_{\mathrm{c}} K$ such that

$$
K^{(i)} \leq H_{1} \times H_{i+1} \times H_{i+2} \times \cdots \times H_{m} \quad \text { and } \quad K^{(i)} \pi_{1} \leq_{\mathrm{o}} H_{1} .
$$

Then we take $K^{(m)}$ for $K_{1}$ and the proof is complete.

Note that $K^{(1)}=[K, K]$ satisfies the relevant conditions, because $H_{1}=K \pi_{1}$ is just infinite and non-abelian. Now suppose that for $i \in\{2, \ldots, m\}$ the group $K^{(i-1)}$ is already available and build $K^{(i)}$ as follows. Let $F=F_{d}$ denote the free profinite group on $d$ generators $a_{1}, \ldots, a_{d}$ and define profinite presentations

$$
1 \rightarrow R_{i} \rightarrow F \stackrel{\varphi_{i}}{\rightarrow} H_{i} \rightarrow 1, \quad 1 \leq i \leq m,
$$

with $a_{j} \varphi_{1}=h_{j}$ for $1 \leq j \leq d$.

Recall that $F / R_{1} \cong H_{1}$ and $F / R_{i} \cong H_{i}$ are non-isomorphic just infinite groups. This gives $R_{i} \nsubseteq R_{1}$, and $R_{i} R_{1} / R_{1}$ is a non-trivial closed normal subgroup of $F / R_{1} \cong H_{1}$. This implies $R_{i} \varphi_{1} \unlhd_{\mathrm{o}} H_{1}$, and we obtain $R_{i} \varphi_{1} \cap K^{(i-1)} \pi_{1} \leq_{\mathrm{o}} K^{(i-1)} \pi_{1}$. By Lemma 2.2, there exists $x_{i-1} \in K^{(i-1)}$ such that $R_{i} \varphi_{1} \cap K^{(i-1)} \pi_{1} \not \leq \mathrm{C}_{K^{(i-1)} \pi_{1}}\left(x_{i-1} \pi_{1}\right)$.

Consequently, we find a word $w_{i} \in R_{i}$ such that $w_{i}\left(h_{1}, \ldots, h_{d}\right) \notin$ $\mathrm{C}_{K^{(i-1)} \pi_{1}}\left(x_{i-1} \pi_{1}\right)$. Using the properties of $K^{(i-1)}$ and $w_{i} \in \operatorname{ker}\left(\pi_{i}\right)$, it follows that $z_{i}=\left[w_{i}\left(h_{1}^{*}, \ldots, h_{d}^{*}\right), x_{i-1}\right] \in K$ satisfies

$$
z_{i} \equiv b \quad\left(\bmod H_{i+1} \times \cdots \times H_{m}\right), \quad \text { where } 1 \neq b \in H_{1} .
$$


Set $K^{(i)}=\overline{\left\langle z_{i}\right\rangle^{K}} \leq_{\mathrm{c}} K$. Visibly $z_{i} \in H_{1} \times H_{i+1} \times \cdots \times H_{m}$, hence $K^{(i)} \leq$ $H_{1} \times H_{i+1} \times \cdots \times H_{m}$. Moreover, $K^{(i)} \pi_{1}=\overline{\langle b\rangle^{H_{1}}}$ is a non-trivial closed normal subgroup of the just infinite group $H_{1}$, so $K^{(i)} \pi_{1} \leq_{\mathrm{o}} H_{1}$.

Acknowledgements. A weak version of Theorem A formed part of the second author's PhD thesis, Royal Holloway, University of London, 2015. We thank the referee for encouraging us to state Proposition 3.2 as a separate result.

\section{REFERENCES}

[1] Yiftach Barnea. Generators of simple Lie algebras and the lower rank of some pro-p-groups. Comm. Algebra, 30(3):1293-1303, 2002.

[2] John D. Dixon, Marcus P. F. du Sautoy, Avinoam Mann, and Dan Segal. Analytic pro-p groups, volume 61 of Cambridge Studies in Advanced Mathematics. Cambridge University Press, Cambridge, 1999.

[3] Mikhail Ershov and Andrei Jaikin-Zapirain. Groups of positive weighted deficiency and their applications. J. Reine Angew. Math., 677:71-134, 2013.

[4] Gundel Klaas, Charles R. Leedham-Green, and Wilhelm Plesken. Linear pro-pgroups of finite width, volume 1674 of Lecture Notes in Mathematics. SpringerVerlag, Berlin, 1997.

[5] Masatake Kuranishi. On everywhere dense imbedding of free groups in Lie groups. Nagoya Math. J., 2:63-71, 1951.

[6] Michel Lazard. Groupes analytiques p-adiques. Inst. Hautes Études Sci. Publ. Math., (26):389-603, 1965.

[7] Alexander Lubotzky and Avinoam Mann. Powerful $p$-groups. II. $p$-adic analytic groups. J. Algebra, 105(2):506-515, 1987.

[8] Alexander Lubotzky and Aner Shalev. On some $\Lambda$-analytic pro- $p$ groups. Israel J. Math., 85(1-3):307-337, 1994.

[9] Colin D. Reid. On the structure of just infinite profinite groups. J. Algebra, 324(9):2249-2261, 2010.

[10] Matteo Vannacci. On hereditarily just infinite profinite groups obtained via iterated wreath products. J. Group Theory, 19(2):233-238, 2016.

Mathematisches Institut Der Heinrich-Heine-Universität, UniverSITÄTsSTR. 1, 40225 DÜSSELDORF, GERMANY

E-mail address: klopsch@math.uni-duesseldorf.de

MAThematisches Institut DeR HeinRICH-Heine-Universität, UniverSITÄtsstr. 1, 40225 DüsseldorF, Germany

E-mail address: vannacci@math.uni-duesseldorf.de 